L. Brown, P. M. Gauthier and W. Hengartner

Nagoya Math. J.

Vol. 57 (1975), 49-58

\title{
CONTINUOUS BOUNDARY BEHAVIOUR FOR FUNCTIONS DEFINED IN THE OPEN UNIT DISC
}

\author{
LEON BROWN ${ }^{1)}$, P. M. GAUTHIER ${ }^{2)}$ AND \\ WALTER HENGARTNER ${ }^{3)}$
}

This paper deals with cluster sets. While cluster sets can be considered in a more abstract setting, we shall limit ourselves to the study of functions $f$ defined in the open unit disc $D$ of the complex plane and taking their values on the Riemann sphere $\overline{\boldsymbol{C}}$. For $p$ a point of the unit circle $C$, we denote by $C(f, p)$ the cluster set of $f$ at $p$, i.e., the set of all values $w \in \overline{\boldsymbol{C}}$ for which there is a sequence $\left\{z_{n}\right\}, z_{n} \in D$, such that $z_{n}$ $\rightarrow p$ and $f\left(z_{n}\right) \rightarrow w$. The point $p$ is called a point of determination for $f$ if $C(f, p)$ is a singleton. In Section 1 we characterize the set of points of determination of a function $f$ defined in $D$. Namely, it is shown that the set of points of determination is a $G_{\delta}$ set, and conversely that given any $G_{\delta}$ set $E$ on $C$, there exists a bounded holomorphic function $f$ on $D$, whose set of points of determination is precisely $E$. We then consider the class of functions meromorphic in $D$ which have the property that each point of the unit circle is a point of determination, or what is equivalent the class of functions continuous from $\bar{D}$ to $\bar{C}$ and meromorphic in $D$.

In Section 2 we fix $f$ and consider $C(f, p)$ as a set-valued function of the variable $p$. From a theorem of M. K. Fort we conclude that $C(f, \cdot)$ is continuous on a residual $G_{\delta}$ set of the unit circle. Conversely, given any residual $G_{\delta}$ set $E$ on $C$, there exists a bounded holomorphic function $f$ such that $E$ is precisely the set of points of continuity of $C(f, \cdot)$.

Received August 10, 1972.

1) Supported in part by the National Science Foundation, Grant GP 20150.

2) Supported by NRC of Canada, Grant A-5597, and a grant from the Gouvernement du Québec.

3) Supported by NRC of Canada, Grant A-7339, and a grant from the Gouvernement du Québec. 
In Section 3 we consider the cluster set as a set-valued function of the variable $f$.

Let $P(D)$ denote the class of all subsets of $D$. For $f$ defined from $D$ to $\bar{C}$ and for $A \in P(D)$, let $C(f, A)$ denote the restricted cluster set of $f$ on $A$, i.e., the set of all values $w \in \bar{C}$ for which there is a sequence $\left\{z_{n}\right\}, z_{n} \in A$, such that $\left|z_{n}\right| \rightarrow 1$ and $f\left(z_{n}\right) \rightarrow w$. In Section 4 we exhibit a "fairly natural" topology on $P(D)$ with the property that $C(f, \cdot)$ is continuous on $P(D)$ if and only if $f$ is normal.

We extend our thanks to the Canadian Mathematical Congress Summer Institute where much of the present research was conducted. It is also a pleasure to thank Takashi Ito for kindly allowing us to quote one of his unpublished results.

\section{Preliminaries.}

We consider the sphere $\bar{C}$ to be endowed with the usual metric $\chi$. A function taking values in $\bar{C}$ will be said to be spherically continuous or $\chi$-continuous if it is continuous with respect to the $\chi$-topology on $\overline{\boldsymbol{C}}$. Let $F(\bar{C})$ denote the space of closed subsets of $\bar{C}$ endowed with the Hausdorff topology. We say that a function $h$ from a topological space $X$ to $F(\bar{C})$ is upper semicontinuous at a point $x \in X$ if for each $\varepsilon>0$ there is a neighbourhood $V$ of $x$ such that

$$
h(y) \subset G(h(x), \varepsilon), \quad \text { for each } y \in V,
$$

where

$$
G(h(x), \varepsilon)=\{w \in \bar{C}: \chi(w, h(x))<\varepsilon\} .
$$

The function $h$ is said to be lower semicontinuous at $x$ if for each $\varepsilon>0$, there is a neighbourhood $V$ of $x$ such that

$$
h(x) \subset G(h(y), \varepsilon), \quad \text { for each } y \in V .
$$

It follows that $h$ is continuous at $x$ if and only if it is both upper and lower semicontinuous at $x$.

Let $\lim \sup _{t \rightarrow x} h(t)$ be the set of all $w \in \bar{C}$ such that for each neighbourhood $U$ of $w$ and each neighbourhood $V$ of $x$, there is a point $y$ in $V$ such that $h(y)$ meets $U$. Let $\lim \inf _{t \rightarrow x} h(t)$ be the set of all $w \in \overline{\boldsymbol{C}}$ such that for each neighbourhood $U$ of $w$, there is a neighbourhood $V$ of $x$ such that $h(y)$ meets $U$, for each $y$ in $V$. 
THEOREM 1. Let $h$ be as above. Then $h$ is lower semicontinuous at $x$ if and only if

a)

$$
\liminf _{t \rightarrow x} h(t) \supset h(x),
$$

and $h$ is upper semicontinuous at $x$ if and only if

b)

$$
h(x) \supset \limsup _{t \rightarrow x} h(t) .
$$

Proof. We prove only that if a) holds then $h$ is lower semicontinuous at $x$. The other statements are straightforward.

Suppose then that a) holds and $\varepsilon>0$. Then for each $w \in h(x)$, there is a neighbourhood $V(w)$ of $x$ such that

$$
h(y) \cap G(w, \varepsilon) \neq \emptyset, \quad \text { for each } y \in V(w) .
$$

It follows that

$$
w \in G(h(y), \varepsilon), \quad \text { for each } y \in V(w) .
$$

Let $G\left(w_{j}, \varepsilon\right), j=1, \cdots, n$, be a finite subcover of $h(x)$ extracted from $\{G(w, \varepsilon): w \in h(x)\}$, and let $V(1), V(2), \cdots, V(n)$, be the corresponding $V(w)$ 's. Then for $V=V(1) \cap V(2) \cap \cdots \cap V(n)$,

$$
h(x) \subset G(h(y), 2 \varepsilon), \quad \text { for each } y \in V .
$$

Hence $h$ is lower semicontinuous at $x$, and the proof is complete.

CoRollary. $h$ is continuous at $x$ if and only if

$$
\liminf _{t \rightarrow x} h(t)=\limsup _{t \rightarrow x} h(t)=h(x) .
$$

We remark that we are dealing with a very special set-valued function, one whose values are closed subsets of the Riemann sphere. For general set-valued functions M. Dollinger has provided a counter-example to show that the above corollary does not hold [6].

One of our principal results will be based on the following.

THEOREM (Fort [7]). If a function from a topological space to $F(\overline{\boldsymbol{C}})$ is upper semicontinuous, then it is continuous at each point of a residual $G_{\delta}$ set. 


\section{Points of determination.}

Let $f$ be a function from $D$ to $\bar{C}$ and let $E$ denote the set of points of determination of $f$. It is readily seen that $E$ is a $G_{\delta}$ set. Indeed, the set of points of indetermination is precisely

$$
\bigcup_{n=1}^{\infty} \bigcap_{s>0}\{p \in C: \omega(f, p, s) \geqq 1 / n\},
$$

where

$$
\omega(f, p, s)=\sup \left\{\chi\left(f(z), f\left(z^{\prime}\right)\right): z, z^{\prime} \in D ;|p-z|<s,\left|p-z^{\prime}\right|<s\right\} .
$$

Thus $C \backslash E$ is an $F_{\sigma}$ set, and so $E$ is a $G_{\delta}$ set. Our first theorem shows that the sets of points of determination are characterized as the $G_{\delta}$ sets.

THEOREM 2. Let $E$ be a $G_{\delta}$ set on the unit circle $C$. Then there exists a function $f$, bounded and holomorphic on $D$, for which $E$ is precisely the set of points of determination.

Proof. Since $E$ is a $G_{\delta}$ set, we may write

$$
C \backslash E=\bigcup_{n=1}^{\infty} F_{n},
$$

where $F_{n}$ is closed and $F_{n} \subset F_{n+1}$ for $n=1,2, \ldots$ Let $B_{n}$ be a Blaschke product whose zeros cluster precisely on $F_{n}$. Thus the set of points of determination of $B_{n}$ is $C \backslash F_{n}$. The function we require is

$$
f=\sum_{n=1}^{\infty} \frac{B_{n}}{3^{n}} .
$$

An important class of functions is the disc algebra, that is, the class $A$ of functions holomorphic in $D$ and continuous on $\bar{D}$. From the point of view of cluster sets (but not of function algebras) it seems natural to consider the more general class of functions holomorphic in $D$ and continuous from $\bar{D}$ to $\bar{C}$. Moreover we may as well allow $f$ to be meromorphic in $D$. Let $A(\chi)$ denote this class, namely the functions continuous from $\bar{D}$ to $\bar{C}$ and meromorphic in $D$. It is easy to see that $A(\chi)$ is just the class of meromorphic function in $D$, for which every point of $C$ is a point of determination.

Very few of the disc-algebra techniques can be utilized to study $A(\chi)$, for unlike $A, A(\chi)$ has no algebraic structure. Indeed, the function 
$\exp [(z+1) /(z-1)]$ is not in $A(\chi)$, yet we can write it as the product of functions in $A(\chi)$,

$$
\exp \left(\frac{z+1}{z-1}\right)=\left[(1-z) \exp \left(\frac{z+1}{z-1}\right)\right](1-z)^{-1},
$$

and as the sum of functions in $A(\chi)$,

$$
\exp \left(\frac{z+1}{z-1}\right)=\left[\exp \left(\frac{z+1}{z-1}\right)+(1-z)^{-1}\right]-(1-z)^{-1}
$$

Thus $A(\chi)$ is closed under neither multiplication nor addition.

We know that the functions in $A$ are characterized as the uniform limits on $\bar{D}$ of polynomials. A similar characterization holds for $A(\chi)$. Clearly the uniform spherical limit on $\bar{D}$ of polynomials is either identically infinite or a holomorphic function in $A(\chi)$. Conversely if $f \in A(\chi)$ is holomorphic, then $f_{r} \rightarrow f$ spherically uniformly, where $f_{r}(z)=f(r z)$, $r<1$. Now $f_{r}$ is the uniform limit of polynomials and hence $f$ is itself the spherically uniform limit of polynomials on $\bar{D}$.

Similarly the spherically uniform limit on $\bar{D}$ of rational functions is either identically infinite or a function in $A(\chi)$. Conversely if $f \in A(\chi)$, then $f_{r} \rightarrow f$ spherically uniformly as before. Now $f_{r}$ is meromorphic on $\bar{D}$, and so $f_{r}$ is the uniform limit of rational functions on $\bar{D}$. Thus $f$ is itself the spherically uniform limit of rational functions on $\bar{D}$.

Another property of the disc algebra $A$ is the following:

THEOREM (Rudin [15]). Let $E$ be a closed set of measure zero on the unit circle $C$, and let $F$ be any continuous (complex-valued) function on $E$. Then there exists a function $f$ in $A$ whose restriction to $E$ is $F$. In fact, we may choose $f$ so that

$$
\sup _{z \in D}|f(z)|=\sup _{z \in E}|F(z)| \text {. }
$$

This result also holds for the class $A(\chi)$. Let $E$ be a closed set of measure zero on $C$ and $P$ spherically continuous on $E$, that is, continuous from $E$ to $\overline{\boldsymbol{C}}$. We divide $E$ into two sets

$$
E_{1}=\{p \in E:|F(p)| \geq 1\}, \quad E_{2}=\{p \in E:|F(p)| \leq 1\} .
$$

Set $F_{1}=1 / F$ on $E_{1}$. Then $F_{1} \neq 0$ on $E_{1} \cap E_{2}$. We can easily construct a continuous extension $\tilde{F}_{1}$ of $F_{1}$ to all of $C$ such that $\tilde{F}_{1} \neq 0$ on $C \backslash E_{1}$. By Rudin's theorem there is a function $f_{1}$ in the disc algebra which 
agrees with $\tilde{F}_{1}$ on $E$. Also there is a function $f_{2}$ in the disc algebra which is equal to zero on $E_{1}$ and equal to $F-1 / f_{1}$ on $E_{2}$. Now set $f=1 / f_{1}+f_{2}$. Thus Rudin's theorem holds also for the class $A(\chi)$. It would be of interest to know whether we can find an $f$ which is holomorphic and has the same properties.

Functions in class $A(\chi)$ are quite tame. More specifically such functions are always normal, as can be seen in several ways. Less obvious, is the fact that such functions are of bounded characteristic [8] and [9].

Although $A(\chi)$ is a very nice class, we shall establish the existence of a function $f \in A(\chi)$, which maps the unit circle $C$ onto $\bar{C}$. Before doing this we recall that Salem and Zygmund [14], and Piranian, Titus and Young [13] have exhibited functions in the disc algebra, which map $C$ onto a square. Their methods do not seem to generalize to $A(\chi)$, however an unpublished technique due to Takashi Ito does. Let $E$ be a Cantor set of zero measure on $C$, and let $\phi$ be the Cantor function mapping $E$ continuously onto the unit interval $[0,1]$. Now let $g$ map $[0,1]$ continuously onto the closed disc, and set $F=g \circ \phi$. By Rudin's theorem there is a function $h \in A$ which agrees with $F$ on $E$. Then $h$ maps $C$ onto the disc. In fact we may choose $h$ such that $\|h\|_{\infty}=1$. Thus $|h(z)|<1$ for $|z|<1$, and so if we set

$$
f(z)=\frac{h(z)}{(1-h(z))^{2}}
$$

we obtain an example of a holomorphic function $f$ with spherically continuous boundary values such that $f$ maps $C$ onto $\bar{C}$. We observe that it is also possible to obtain such an example by mapping $D$ onto a Riemann surface whose "border" projects onto the Riemann sphere.

\section{Cluster set as a function on the boundary.}

Let $f$ be a fixed function from $D$ to $\overline{\boldsymbol{C}}$. Then the cluster set $C(f, p)$ becomes a function of the variable $p$ and taking values in the space $F(\bar{C})$ of closed subsets of $\overline{\boldsymbol{C}}$. One easily shows:

LEMMA 1. For an arbitrary function $f$ from $D$ to $\overline{\boldsymbol{C}}$, the cluster set $C(f, \cdot)$ is an upper semi-continuous function on $C$.

We note that if $p$ is a point of determination for $f$ then $C(f, \cdot)$ is necessarily continuous at $p$ but not conversely. 
THEOREM 3. The set of points at which $C(f, \cdot)$ is continuous is a residual $G_{\delta}$ set on $C$. Moreover if $E$ is any residual $G_{\delta}$ set on $C$, then there is a bounded holomorphic function $f$ on $D$ for which $E$ is precisely the set of points of continuity for $C(f, \cdot)$.

Proof. The first part follows from Fort's theorem and Lemma 1.

Let $E$ be a residual $G_{\delta}$ set in $C$. Then by Theorem 2 there is a bounded holomorphic function $f$ for which $E$ is precisely the set of points of determination. Thus $C(f, \cdot)$ is continuous at each point of $E$. If $p \in C \backslash E$, then $C(f, p)$ is not a singleton, but since $E$ is dense, $p$ is the limit of points at which the cluster set is a singleton. Hence $C(f, \cdot)$ is not continuous at $p$. This solves the problem raised by Koo [11] of characterizing the set of points of continuity of $C(f, \cdot)$.

\section{Cluster set as a function of functions.}

Instead of fixing $f$ and varying $p$, we could fix $p$ and vary $f$. Thus $C(\cdot, p)$ becomes a function of the variable $f$. Actually what we shall do is consider the global cluster set $C(f)$ as a function of $f$. It will be clear as we proceed that whatever we conclude concerning the function $C(f)$ also holds for the local cluster set $C(f, p)$ considered as a function of $f$. We recall that $C(f)$ is the set of all values $w \in \bar{C}$ for which there is a sequence $\left\{z_{n}\right\}, z_{n} \in D$, such that $\left|z_{n}\right| \rightarrow 1$ and $f\left(z_{n}\right) \rightarrow w$.

Let $\bar{C}^{D}$ denote the set of all functions from $D$ to $\bar{C}$. We consider $\bar{C}^{D}$ to be endowed with the sup topology:

$$
d_{1}(f, g)=\sup _{D} \chi(f(z), g(z)), \quad f, g \in \bar{C}^{D} .
$$

By the methods of Colwell [5], it can be shown that the global cluster set is a continuous function from $\left(\bar{C}^{D}, d_{1}\right)$ into $F(\bar{C})$.

Actually a slightly stronger result is true. For $f, g \in \bar{C}^{D}$, we write

$$
d(f, g)=\limsup _{|z| \rightarrow 1} \chi(f(z), g(z)) .
$$

In case $f$ and $g$ are $\chi$-continuous, this is equivalent to taking the sup on the ideal boundary of the Stone-Čech compactification. It is clear that the topology enduced by $d$ is coarser than that induced by $d_{1}$ and the global cluster set is continuous on $\left(\bar{C}^{D}, d\right)$. Moreover, if $A$ is any subset of $D$, then $C(\cdot, A)$ is still a continuous function on $\left(\bar{C}^{D}, d\right)$.

An interesting question is whether this induces a metric topology. 
This question can be phrased as a uniqueness problem: Suppose $f$ and $g$ are meromorphic in $D$ and

$$
\limsup _{|z| \rightarrow 1} \chi(f(z), g(z))=0 \text {. }
$$

Does this imply that $f \equiv g$ ? J. Clunie [3] has answered this question in the negative by showing that there exist distinct meromorphic functions satisfying (3) and more recently $H$. Allen [1] and P. Lappan (to appear) have exhibited holomorphic functions with the same property.

Thus far we have considered two topologies on functions. Consider now the space $M(D)$, of meromorphic functions on $D$ to be endowed with either the topology of uniform convergence on compact subsets or of spherically uniform convergence on compact subsets. The cluster set may be neither upper nor lower semicontinuous. For example if $f_{n}(z)$ $=z^{n}$, then $f_{n} \rightarrow 0$ but $C\left(f_{n}\right)=C$ and $C(0)=\{0\}$. Thus $C(\cdot)$ is neither upper nor lower semicontinuous at $f \equiv 0$.

However we can show the following: The set of functions in $M(D)$ at which the cluster set is upper semicontinuous is precisely the set $M_{0}$ of functions at which the cluster set is total (equal to $\overline{\boldsymbol{C}}$ ), and $M_{0}$ is a residual set. Firstly if the cluster set is total at $f$, it is clear that the cluster set is upper semicontinuous at $f$. The methods of Kierst and Szpilrajn [10] easily establish that $M_{0}$ is residual in $M(D)$ and, in particular, dense. If the cluster set at $f$ is not total, then the cluster set cannot be upper semicontinuous at $f$ since there are functions in every neighbourhood of $f$ at which the cluster set is total. So much for upper semicontinuity.

It can be shown that the cluster set is lower semicontinuous at no function in $M(D)$. Indeed let $f \in M(D)$. Choose $w_{0} \in C(f)$ and $\varepsilon, \delta>0$. Also, choose $w_{1}$ finite and such that $\chi\left(w_{1}, w_{0}\right)=2 \varepsilon$. Let $N(f, K, \delta)$ be a basic open neighbourhood of $f$ where $K \subset D$ is compact. Let $p_{n}$ be the principal part of $f$ on $K_{n}$. By Runge's Theorem, there is a rational function $r(z)$ such that

$$
\left\|r-\left(f-p_{n}\right)\right\|_{K}<\delta
$$

and

$$
\chi\left(r(z)+p_{n}(z), w_{1}\right)<\varepsilon, \quad z \in C .
$$

Now set $g=r+p_{n}$. Then $g \in N(f, K, \delta)$, but $C(f) \not \subset G(C(g), \varepsilon)$. Since 
$\delta$ may be chosen arbitrarily small, the cluster set is not lower semicontinuous at $f$. This complete the proof.

\section{Cluster set as a function of sets.}

In this section $A$ will denote a subset of $D$ and $C(f, A)$ the cluster set of $f$ restricted to $A$ as defined earlier. Also we recall that $P(D)$ denotes the class of all subsets of $D$, and $\rho$ denotes the non-Euclidean hyperbolic metric on $D$.

For $A \subset D$, let $A$ denote the (relative) closure of $A$ in $D$, and set

$$
G_{\rho}(A, s)=\{z \in D: \rho(z, A)<s\}, \text { where } s>0 .
$$

If $A_{1}, A_{2} \subset D$, we write

$d_{r}\left(A_{1}, A_{2}\right)=\inf \left\{s:\left[A_{1} \cap(|z|>r)\right] \subset G_{p}\left(A_{2}, s\right),\left[A_{2} \cap(|z|>r)\right] \subset G_{p}\left(A_{1}, s\right)\right\}$.

Also, set

$$
d\left(A_{1}, A_{2}\right)=\lim _{r \rightarrow 1} d_{r}\left(A_{1}, A_{2}\right)
$$

and

$$
\sigma\left(A_{1}, A_{2}\right)=\left\{\begin{array}{l}
\frac{d\left(A_{1}, A_{2}\right)}{1+d\left(A_{1}, A_{2}\right)}, \quad \text { if } d\left(A_{1}, A_{2}\right) \neq \infty \\
1, \text { otherwise. }
\end{array}\right.
$$

Now $\sigma$ is a pseudometric on the class $P(D)$. Thus, for fixed $f, C(f, \cdot)$ can be thought of as a function from the topological space $P(D)$ into the topological space $F(\bar{C})$. Following Lappan [12], we define a function $f: D \rightarrow \overline{\boldsymbol{C}}$ to be normal if and only if it is uniformly $\rho-\chi$ continuous. The following generalizes a result of Cima and Rung [2].

THEOREM 4. Let $f$ be a function from $D$ to $\overline{\boldsymbol{C}}$. Then $C(f, \cdot)$ is continuous from $P(D)$ to $F(\bar{C})$ if and only if $f$ is normal.

The proof is straightforward and we omit it.

\section{REFERENCES}

[1] Allen, H.: Distinct holomorphic functions with idetical boundary values (preprint).

[ 2 ] Cima, J. A., Rung, D. C.: Normal functions and a class of associated boundary functions. Israel J. Math. 4 (1966), 119-126. MR 34 \#6019.

[ 3 ] Clunie, J.: On a problem of Gauthier. Mathematika 18 (1971), 126-139. 
[4] Collingwood, E. F., Lohwater, A. J.: The theory of cluster sets. Cambridge University Press, Cambridge, 1966. MR 38 \#325.

[5] Colwell, Peter: Cluster set theorems for uniformly convergent sequences of functions. Proc. Amer. Math. Soc. 18 (1967), 48-52. MR 37 \#5398.

[6] Dollinger, M. B.: Some aspects of spectral theory on banach spaces. Doctoral dissertation, University of Illinois, 1968.

[ 7 ] Fort, M. K. Jr.: A unified theory of semi-continuity. Duke Math. J. 16 (1949), 237-246. MR 10-716.

[ 8 ] Gauthier, P. M., Hengartner, Walter: Local harmonic majorants of functions subharmonic in the unit disc. J. Analyse Math. 26 (1973), 405-412.

[9] Heins, Maurice: The boundary values of meromorphic functions having bounded Nevanlinna characteristic. J. Analysis Math. 18 (1967), 121-131. MR 35 \#4401.

[10] Kierst, S., Szpilrajn, E.: Sur certaines singularités des fonctions analytiques uniformes. Fund. Math. 21 (1933), 276-294.

[11] Koo, Shu-Chung: Some properties of functions and cluster sets (unpublished).

[12] Lappan, Peter: Some results on harmonic normal functions. Math. Z. 90 (1965), 155-159. MR 35 \#397.

[13] Piranian, George; Titus, C. J. and Young, G. S.: Conformal mappings and Peano curves. Michigan Math. J. 1 (1952), 69-72. MR 14-262.

[14] Rudin, W.: Boundary values of continuous analytic functions, Proc. A.M.S., 7 (1956), 808-811. MR 18-472.

[15] Salem, Raphael and Zygmund, A.: Lacunary power series and Peano curves. Duke Math. J. 12 (1945), 569-578. MR 7-378.

Wayne State University

Université de Montréal

Université Laval 